\title{
Controlling coexisting attractors of an impacting system via linear augmentation
}

\author{
Yang Liu ${ }^{\mathrm{a}}$, Joseph Páez Chávez ${ }^{\mathrm{b}, \mathrm{c}}$ \\ ${ }^{a}$ College of Engineering Mathematics and Physical Sciences, University of Exeter, Rennes Drive, Exeter EX 4 4RN, UK \\ ${ }^{b}$ Center for Dynamics, Department of Mathematics, TU Dresden, D-01062 Dresden, Germany \\ ${ }^{c}$ Center for Applied Dynamical Systems and Computational Methods (CADSCOM), Faculty of Natural Sciences and \\ Mathematics, Escuela Superior Politécnica del Litoral, P.O. Box 09-01-5863, Guayaquil, Ecuador
}

\begin{abstract}
This paper studies the control of coexisting attractors in an impacting system via a recently developed control law based on linear augmentation. Special attention is given to two control issues in the framework of multistable engineering systems, namely, the switching between coexisting attractors without altering the system's main parameters and the avoidance of grazing-induced chaotic responses. The effectiveness of the proposed control scheme is confirmed numerically for the case of a periodically excited, soft impact oscillator. Our analysis shows how path-following techniques for non-smooth systems can be used in order to determine the optimal control parameters in terms of energy expenditure due to the control signal and transient behavior of the control error, which can be applied to a broad range of engineering problems.
\end{abstract}

Keywords: Multistability; Non-smooth system; Impact oscillator; Linear augmentation; Numerical continuation; Optimal control

\section{Introduction}

Impacting systems are widely found in engineering applications, such as ground moling [1], percussive drilling [2], self-propelled capsule systems [3,4], where the impacting behavior is a part of the original design, or gearboxes [5], bearings [6], and rotor systems [7], where the impact between mechanical elements is an undesired effect due to wear or defective design. A common feature of impacting systems is that of multistability [8], referring to the coexistence of two or more attractors for a given set of parameter values. From a practical point of view, there are two control issues that are frequently studied in the framework of multistable systems. The first one consists in exploiting the fact that such systems can switch between several operation modes without altering the main system parameters. For example, the direction of motion (forward or backward) of a self-propelled vibro-impact capsule system can be controlled by switching between two coexisting attractors, see e.g. $[9,10]$. Another case where multiple coexisting solutions can be advantageous is considered in $[2,11]$, where the efficiency of drilling applications can be improved by choosing suitable operation modes while maintaining a desired rate of penetration. On the other hand, multistability can also be the source of system malfunctions, specially under the presence of perturbations steering the system to an unwanted operation mode, e.g. drill-string failure due to stick-slip oscillations [12-14], rotor-stator impacts due to imbalance [7, 15]. Therefore, the development of reliable control methods in order to switch between coexisting attractors or suppress some certain unwanted multistable states is crucial.

In the present work, we will study the multistability of a periodically excited oscillator with soft impacts. Such systems are very common to a broad range of engineering applications where the repeated collision of mechanical parts is unavoidable, see for example $[5,16,17]$. In many cases, impacting behavior leads to undesired effects, for instance, reduction of the efficiency and operating life of the system, which becomes even more critical in the presence of grazing bifurcations [18-20]. In [21-24], a large number

Email addresses: y.liu2@exeter.ac.uk (Yang Liu), jpaez@espol.edu.ec (Joseph Páez Chávez) 


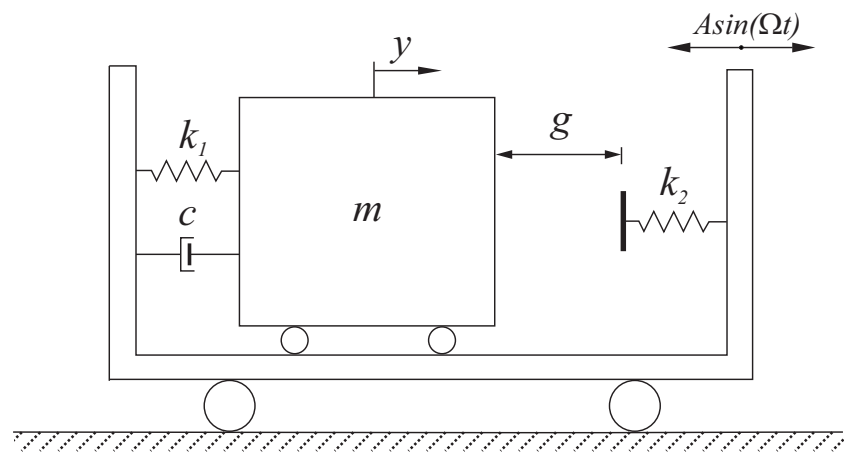

Figure 1: Physical model of the impact oscillator.

of coexisting attractors in an impact oscillator close to grazing has been observed both numerically and experimentally. Pavlovskaia et al. [25] studied several bifurcation scenarios, among which they observed the occurrence of coexisting attractors through a discontinuous transition from one orbit to another via a boundary crisis closely related to grazing events. Various investigations have shown that persistent chaotic motion can be observed in the vicinity of grazing bifurcations in impact oscillators, leading to undesired system operation and sensitivity to small perturbations. This issue has attracted a significant amount of interest in the past. For instance, in [26] the authors proposed a method to control chaotic attractors in an impacting system based on small perturbations of a suitably chosen system parameter. De Souza et al. [27] considered a similar scenario where chaotic motion was avoided via feedback damping control. In [28], a discrete linear feedback control strategy was presented for controlling the persistence of a local attractor near a grazing periodic trajectory in an impact oscillator in the presence of discontinuous jumps.

Our main concern in the present paper is to address the two control issues outlined before, namely, the advantageous use of multistability in order to optimize the operation of an impact oscillator and the avoidance of chaotic responses in the vicinity of grazing events. For these purposes, we will employ a recently developed control method based on linear augmentation, which is achieved by coupling systems via a linear feedback consisting of a simple decaying function [29]. This approach has been shown to be effective in targeting desired steady-state solutions [30], suppressing bistability [31], regulating the dynamics of drive response systems [32], and controlling the dynamics of hidden attractors [33], where the linear augmentation control was used to stabilize fixed points of nonlinear oscillators. In order to switch between coexisting attractors in non-smooth systems without affecting their basins of attraction, we use a modified version of the linear augmentation control method studied in previous investigations. A preliminary work in this direction has been done by Liu et al. [8] for controlling both smooth and non-smooth dynamical systems using an intermittent feedback controller. This control scheme is applied repeatedly in the time domain when a current attractor satisfies a proximity condition with regards to a desired attractor. For the control issue regarding the avoidance of chaotic responses in the vicinity of grazing events, we study in detail the bifurcation scenario of the considered impact oscillator in order to find a suitable periodic solution that can be used as target attractor. A similar approach has been employed in [9] to control the motion of a vibro-impact capsule system using a position feedback controller. By slightly adjusting its position control gain, this controller was able to restrain the chaotic response and significantly enlarge the basin of attraction of the desired solution, thereby increasing the robustness of this operation mode against external perturbations. In this paper we will show how the modified linear augmentation control law can be used to effectively drive the impacting system to the desired periodic solution and, furthermore, how the control scheme can be optimized via the application of path-following techniques for non-smooth systems, implemented via the continuation platform COCO [34].

The main contribution of the present paper is twofold: first, controlling multistability in impacting systems without affecting the system's dynamics, and second, a numerical approach based on pathfollowing techniques to optimize the control scheme in terms of the energy spent by the controller. The modified linear augmentation control used in this paper is implemented through coupling a linear system 
describing the difference in position and velocity between a current and a desired periodic response. The novelty of this approach is that system behavior is not affected by the controller once the system has settled down to the desired operation mode, while the coupling strength is adjusted to optimize energy expenditure and transient times. The rest of the paper is organized as follows. In Section 2, the physical model and equations of motion of the impact oscillator are introduced, as well as the mathematical formulation of the linear augmentation control law. In Section 3, the proposed control method is studied numerically, with special attention on using multistability to suppress grazing-induced chaotic attractors. The performance and optimal operation of the control scheme is analyzed in Section 4. For this purpose, numerical continuation methods are applied in order to investigate the behavior of the energy costs and the error during the transient phase before stabilization. Finally, the main conclusions of the present work are presented in Section 5 .

\section{Mathematical description}

Many mechanical systems experiencing intermittent impacts can be represented by a simple model of the impact oscillator shown in Fig. 1, which will be investigated in the present work. In this model, it is assumed that the discontinuity boundary is neither motion- nor time-dependent but fixed at $x=e$, with $e>0$ being the nondimensional gap (see below). The equations of motion of the impact oscillator shown in Fig. 1 are given in nondimensional form by

$$
\left\{\begin{array}{l}
x^{\prime}(\tau)=v(\tau) \\
v^{\prime}(\tau)=a \omega^{2} \sin (\omega \tau)-2 \zeta v(\tau)-x(\tau)-\beta(x(\tau)-e) H(x(\tau)-e),
\end{array}\right.
$$

where $H(\cdot)$ stands for the Heaviside step function and $x^{\prime}, v^{\prime}$ represent differentiation with respect to the dimensionless time $\tau$. In the governing equations (1), the variables and parameters of the system are nondimensionalized according to:

$$
\begin{aligned}
& \omega_{n}=\sqrt{\frac{k_{1}}{m}}, \quad \tau=\omega_{n} t, \quad \omega=\frac{\Omega}{\omega_{n}}, \quad \zeta=\frac{c}{2 m \omega_{n}}, \\
& x=\frac{y}{y_{0}}, \quad e=\frac{g}{y_{0}}, \quad a=\frac{A}{y_{0}}, \quad \beta=\frac{k_{2}}{k_{1}},
\end{aligned}
$$

where $y_{0}>0$ is an arbitrary reference length, $\omega_{n}$ is the natural frequency, $\omega$ is the frequency ratio, $\zeta$ is the damping ratio, $\beta$ is the stiffness ratio, $e$ is the nondimensional gap between the mass and the secondary spring, and $a$ represents the nondimensionalized amplitude of the external excitation.

In the present work, we will consider a control signal $u(\tau), \tau \geq 0$, which will be applied to the system's external excitation as follows

$$
\left\{\begin{array}{l}
x^{\prime}(\tau)=v(\tau) \\
v^{\prime}(\tau)=\left[a \omega^{2} \sin (\omega \tau)+u(\tau)\right]-2 \zeta v(\tau)-x(\tau)-\beta(x(\tau)-e) H(x(\tau)-e),
\end{array}\right.
$$

where

$$
u(\tau)=\epsilon\left[\left(x_{d}(\tau)-x(\tau)\right)+\lambda\left(v_{d}(\tau)-v(\tau)\right)\right], \quad \tau \geq 0,
$$

defines the linear augmentation control law. In the expression above, $\epsilon$ stands for the coupling strength between the impact oscillator and the linear control system, $\lambda$ is a constant weighting factor, and $\left(x_{d}(\tau), v_{d}(\tau)\right), \tau \geq 0$, gives the position and velocity of the target solution to which the system should be driven. In the absence of coupling $(\epsilon=0)$, system $(3)$ reduces to the original model $(1)$. When the control is activated with some suitable $\epsilon>0$, the current system trajectory $(x(\tau), v(\tau))$ converges to the desired solution $\left(x_{d}(\tau), v_{d}(\tau)\right)$ for $\tau$ sufficiently large (formally, for $\tau \rightarrow \infty$ ), due to which the control signal $u$ decays to zero and the modified system (3) becomes the original model (1). This approach will be used in our investigation in order to drive the impact oscillator from an undesired operation mode, such as chaotic motion or a periodic solution with high power consumption, to a desired periodic behavior, 
without altering the system's parameters. In practice, the current state of the considered system, i.e. the position and velocity of the oscillating mass, can be easily measured in real-time through nondestructive testing, e.g. using eddy current probes. The control signal based on the coupling of the linear control system can be applied through an electrodynamic shaker [21], which provides harmonic excitation of the impact oscillator, or using a linear actuator (e.g. piezoelectric actuator) acting directly on the oscillating mass. The control applied through an electrodynamic shaker has been verified experimentally on the one-degree-of-freedom impact oscillator in [8].

\section{Numerical investigation of the linear augmentation control law}

In this section, we will study the effectiveness of the linear augmentation controller with regards to the two control objectives outlined before, namely, switching between coexisting periodic attractors and suppressing undesired chaotic responses. A major concern in our study will be to optimize the energy spent in the system's operation in the absence of control, therefore we will consider the following quantity

$$
P_{A V G}=\frac{1}{T} \int_{0}^{T} a \omega^{2} \sin (\omega \tau) v(\tau) d \tau
$$

which gives the average power per period used to drive the moving mass, with $T>0$ being the orbital period. This measure will help us identify those periodic solutions for which the energy is used in an efficient manner. In Section 4.1, we will introduce two additional measures that will be used to analyze the performance of the linear augmented controller.
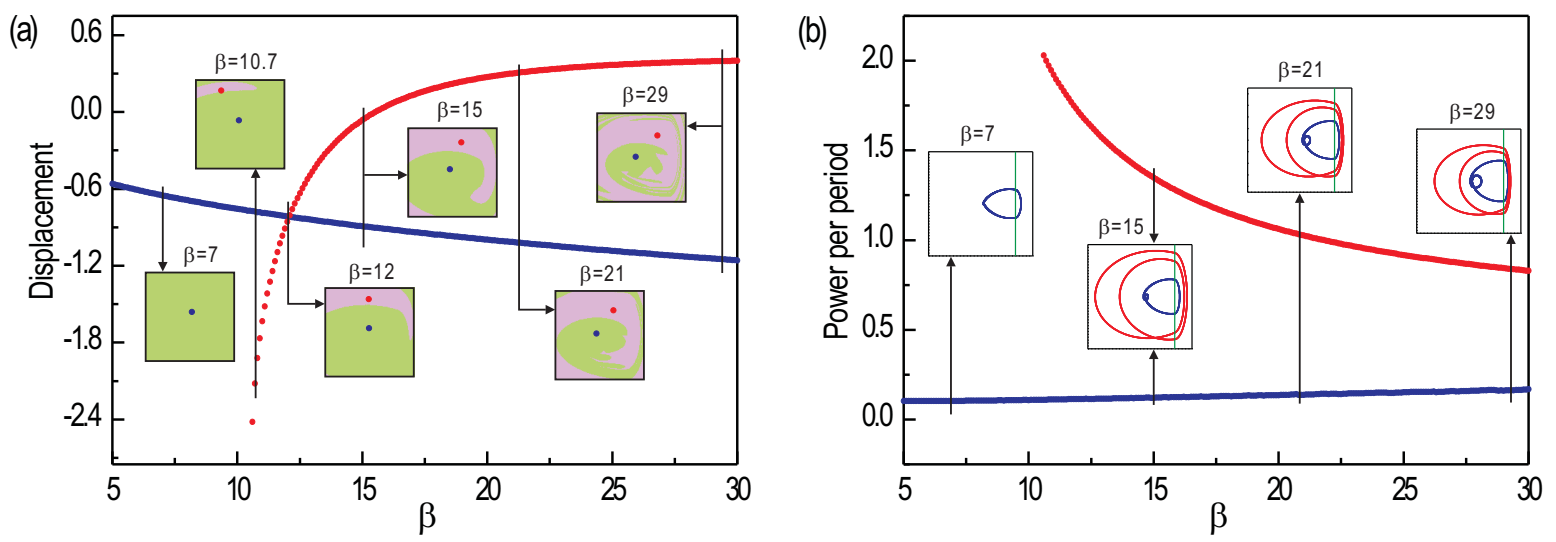

Figure 2: (a) Bifurcation diagram and (b) average power consumption for the impact oscillator, computed for $\zeta=0.01$, $e=1.26, a=2.21, \omega=0.69$ and varying stiffness ratio $\beta$. The period-1 response with one impact per period is denoted by blue dots, and the coexisting period-1 solution with two impacts per period is shown by red dots. Internal diagrams in (a) show the evolution of the basins of attraction of the system, while the inner plots in (b) present the corresponding periodic orbits. The location of the impact boundary is shown by the vertical green line.

\subsection{Controlling multistability}

Our analysis begins with a system configuration for which bistability can be observed, see Fig. 2, where the stiffness ratio varies in the range $\beta \in[5,30]$. Panel (a) presents the bifurcation diagram of the system, where blue dots denote period-1 attractors with one impact per period, red dots represent period-1 attractors with two impacts per period, and the inner diagrams show the evolution of the basins of attraction as $\beta$ varies. It can be seen from this figure that the impact oscillator is monostable for $\beta \in[5,10.6)$, and the period-1 attractor with two impacts emerges at $\beta \approx 10.6$, where the system becomes bistable. As $\beta$ increases, the basin of the period-1 attractor with one impact shrinks, due to 


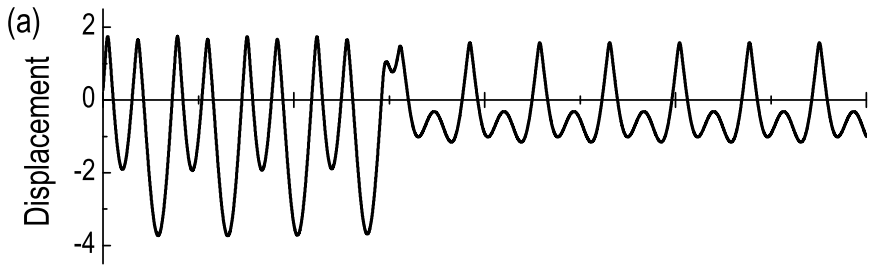

(b)
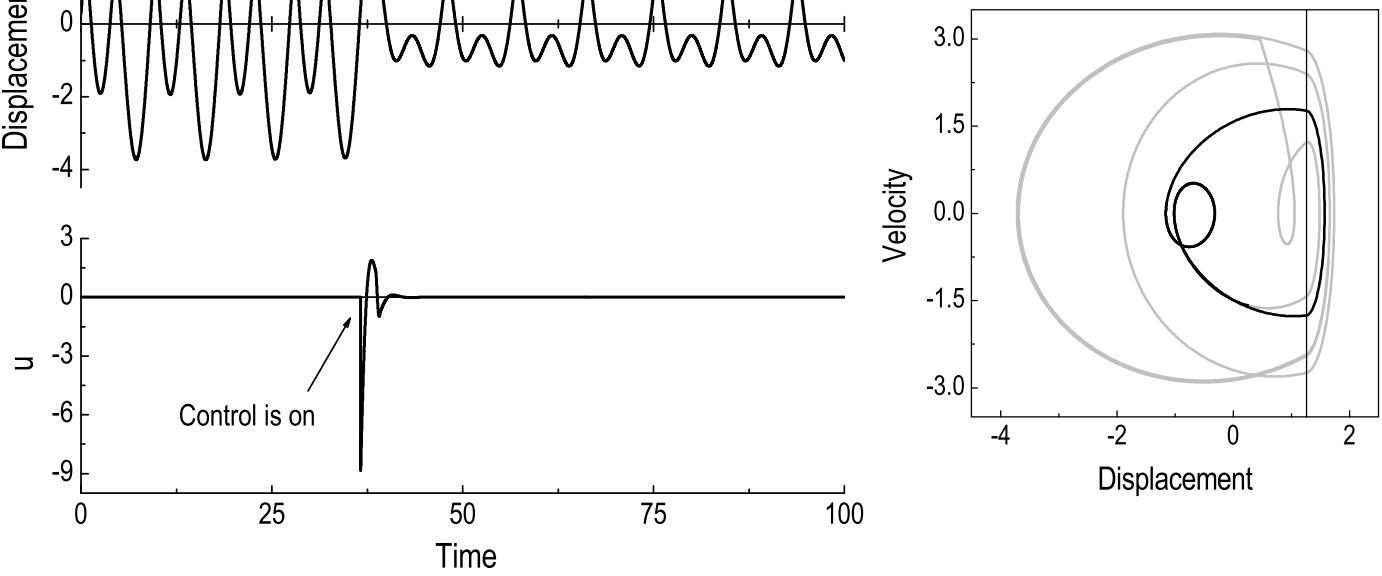

Figure 3: (a) Time history of the mass position of the impact oscillator (upper panel) and the control signal $u$ (lower panel). (b) Phase plot showing the transition from the current solution (grey) to the desired solution (black), computed for $\beta=29$, $\zeta=0.01, e=1.26, a=2.21, \omega=0.69, \epsilon=2$ and $\lambda=1$. The location of the impact boundary is represented by the vertical line in the phase diagram.

which the sensitivity of this solution to perturbations increases and therefore a control mechanism may be required in order to guarantee that the system remains in this mode of operation. The average power dissipated by the system under the two periodic regimes mentioned above is presented in Fig. 2(b), with internal panels showing the corresponding periodic orbits for selected values of stiffness ratio. As can be seen from this figure, the power consumption of the period-1 attractor with two impacts is significantly larger than for the period-1 attractor with one impact. According to this scenario, it becomes evident that maintaining the system on the latter operation mode is crucial to guarantee an efficient use of power. Consider, for instance, a drilling system as that described in [2]. During operation, the hardness of the rock formations usually changes as the drill-bit progresses into the borehole. These changes may induce dynamical scenarios for which multiple stable periodic regimes coexist, and one of the main concerns is to drive the system to those modes where the rate of penetration and the power consumption yield a suitable balance. In our case, we will be therefore interested in driving the system from the undesired state where the power consumption is high to the periodic solution with a lower power dissipation, as shown in Fig. 2(b).

The system response under the linear augmented controller is shown in Fig. 3, which includes time histories of the mass position of the impact oscillator and the control signal $u$. As can be seen from Fig. $3(\mathrm{a})$, the linear augmented control was switched on after five periods of external excitation, at $\tau \approx 36.63$, and the duration of the phase after which the transients have significantly decayed was about 7.81 . In the lower panel of Fig. 3(a), it can be seen that once the system settled on the desired attractor, the control signal decays to zero, due to which the controlled system (3) becomes the original uncontrolled model (1). Panel (b) of Fig. 3 shows the transition from the current solution (grey color) to the desired solution (black color), therefore confirming the effectiveness of the control method in this case. It should be noted, however, that the control signal observed in the lower panel of Fig. 3(a) presents a relatively high peak in comparison to the amplitude of the periodic excitation $a=2.21$, which may pose problems from a practical point of view, as such peaks can damage the control system or the actuators. Furthermore, it is not clear whether the duration of the transient lies within tolerable limits, which may be a crucial factor for certain applications. Therefore, in Section 4 we will analyze in detail the performance of the linear augmented control law via numerical continuation techniques and determine parameter values for which the control can be applied in optimal conditions.

Let us now study the effectiveness of the linear augmentation controller for a scenario where multiple attractors coexist. Fig. 4 shows the basins of attraction for which period-2, period-3, period-5, and period- 

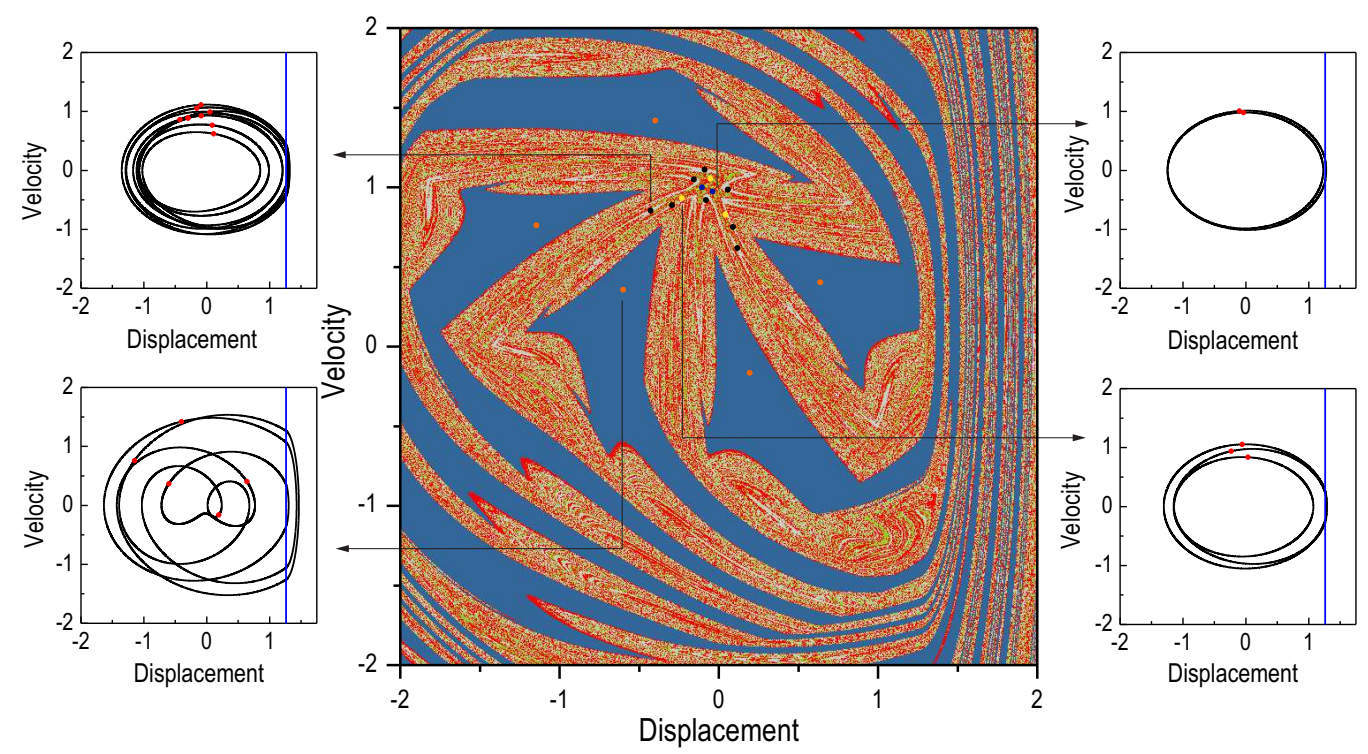

Figure 4: Basins of attraction for the impact oscillator with $\zeta=0.01, e=1.26, a=0.7, \omega=0.8044$, and $\beta=29$. The system possesses in this case four coexisting attractors: the period-2 (blue dots with green basin), the period-3 (yellow dots with red basin), the period-5 (orange dots with blue basin), and the period-8 solutions (black dots with grey basin).

8 solutions coexist. It can be seen from the figure that the basins of attraction are fractally interwoven due to grazing events. These attractors are extremely sensitive to perturbations, due to which a control mechanism for protection against noise-induced basin hopping is always desirable. Here, we will assume that the desired operation mode is given by the period-2 solution shown in Fig. 4 (panel at the top right). Therefore, any perturbation causing a switching to another attractor should be corrected by the linear augmentation controller. The effectiveness of the controller is tested in Fig. 5 for two cases: switching from the period-8 (panel (a)) and switching from the period-5 (panel (b)) solutions to the desired period2 attractor. In both cases the controller was switched on at $\tau \approx 195$, after which the system response converged in short time to the desired period-2 solution. As can be seen from Fig. 5, the controller produces an impulsive perturbation with amplitude significantly greater than the harmonic excitation.
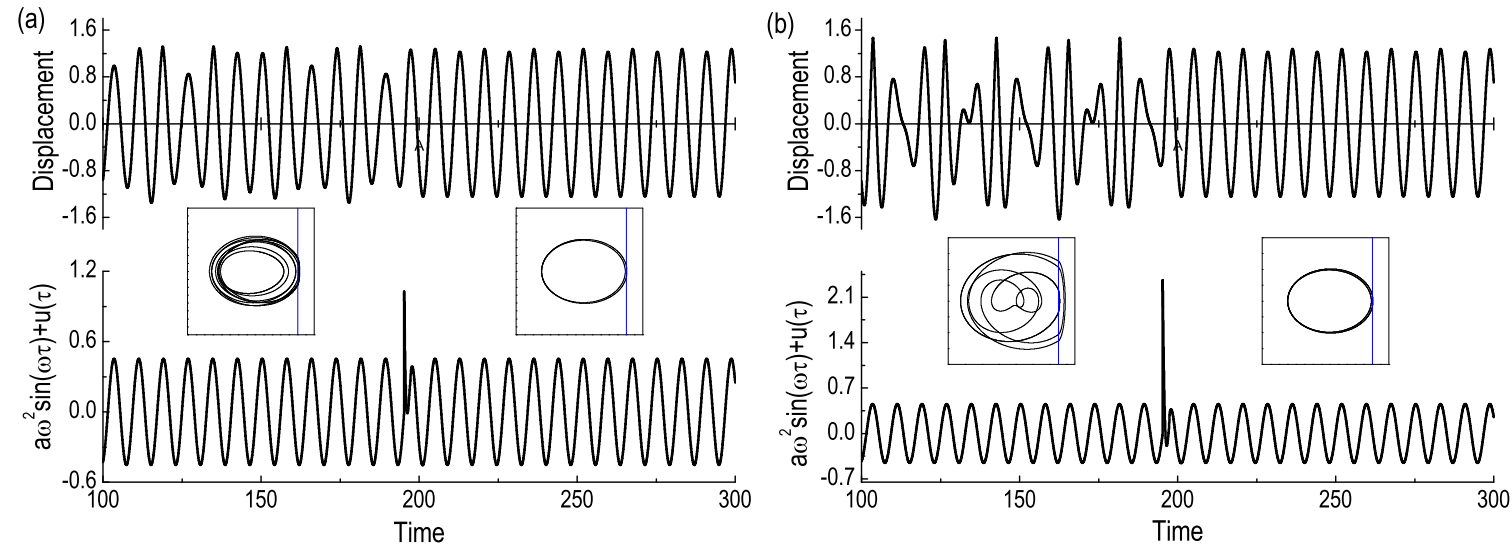

Figure 5: Trajectories and external excitations of the impact oscillator with the linear augmentation control inputs as a function of time, computed for $\zeta=0.01, e=1.26, a=0.7, \omega=0.8044$, and $\beta=29$. The impact oscillator is controlled from (a) the period-8 attractor (denoted by black dots with grey basin in Fig. 4), and (b) the period-5 attractor (marked by orange dots with blue basin in Fig. 4) to the period-2 attractor (denoted by blue dots with green basin in Fig. 4 ). 


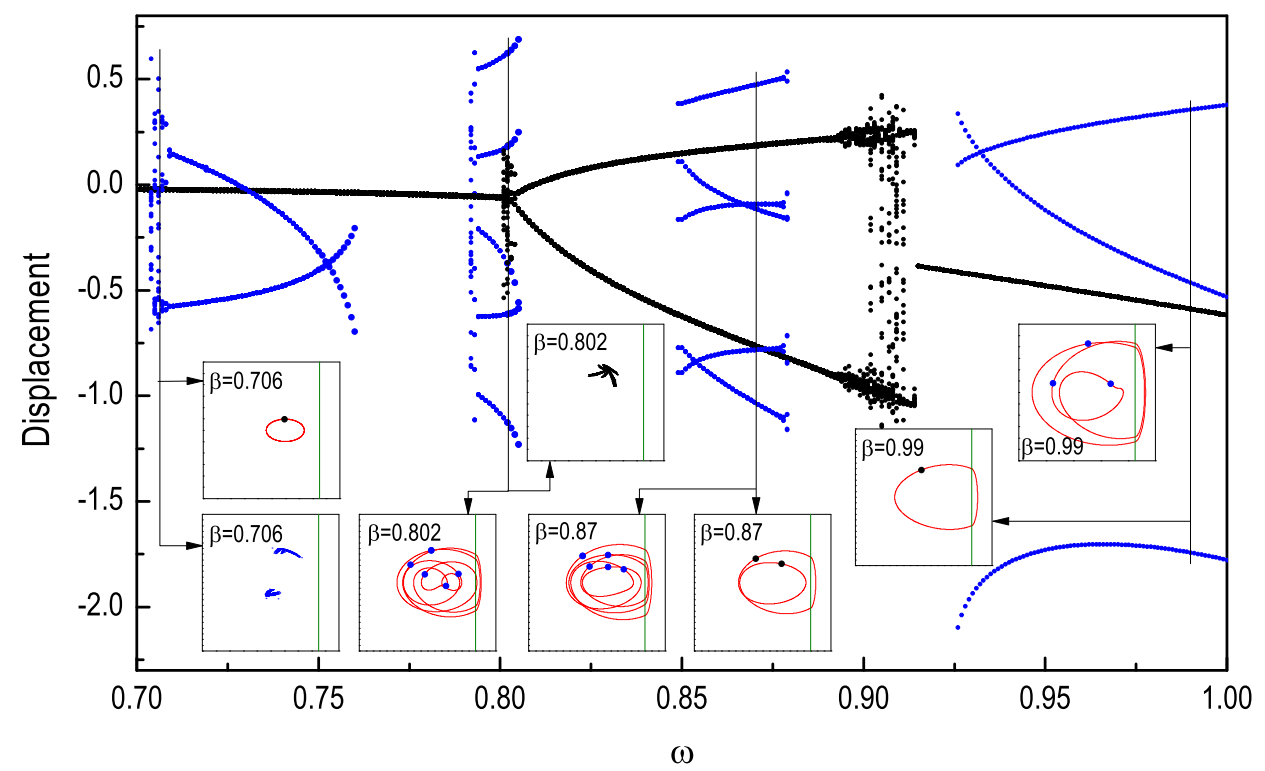

Figure 6: Bifurcation diagram of the impact oscillator with respect to the excitation frequency $\omega$, computed for $\zeta=0.01$, $e=1.26, a=0.7$ and $\beta=28$. Black and blue dots mark the coexisting attractors found during the computation. The inner diagrams depict orbits of the system together with Poincaré sections. The location of the impact boundary is shown by the vertical green line.
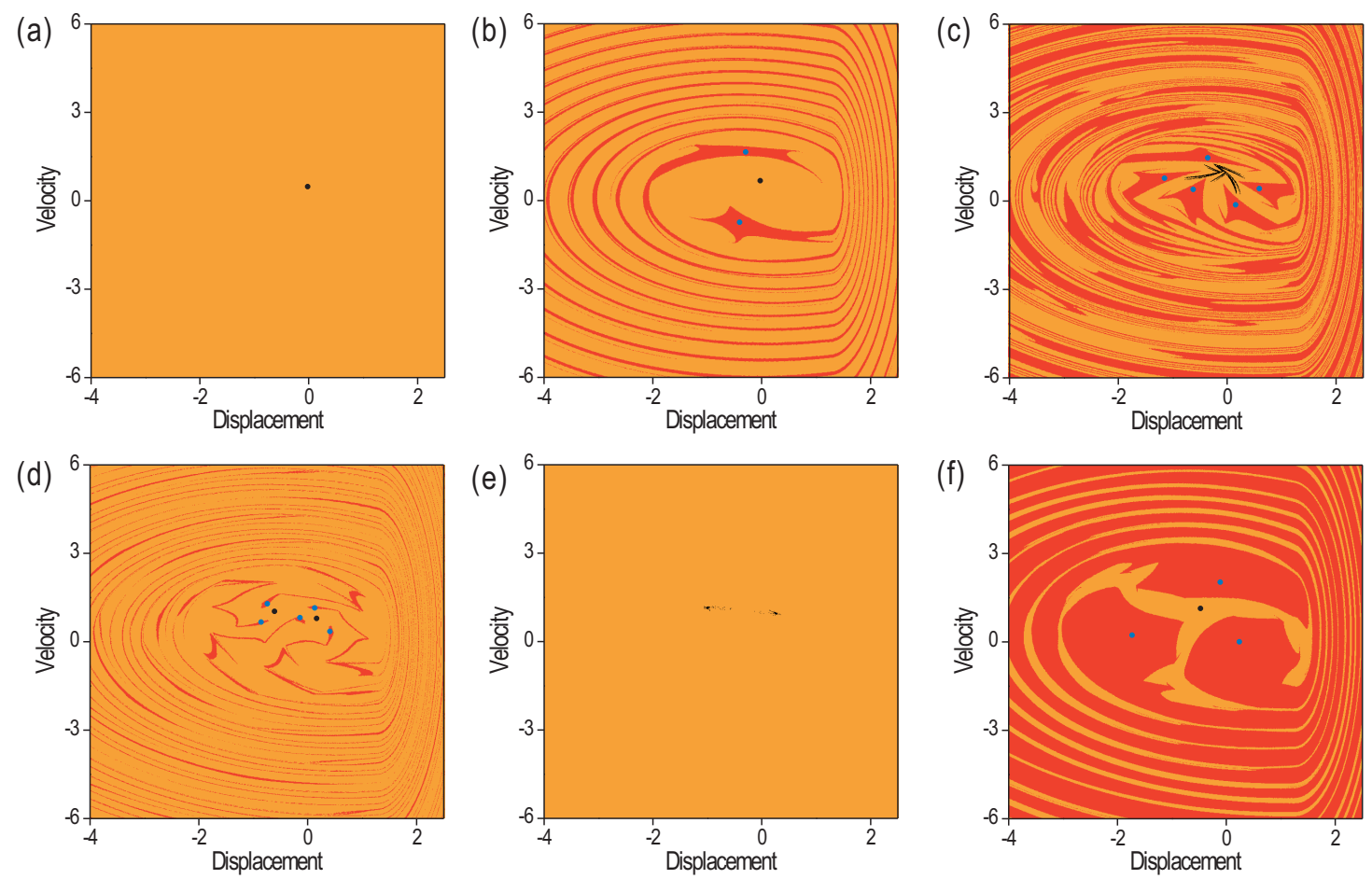

Figure 7: Evolution of the basins of attraction of the impact oscillator computed for $\zeta=0.01, e=1.26, a=0.7, \beta=28$ and (a) $\omega=0.7$, (b) $\omega=0.75$, (c) $\omega=0.802$, (d) $\omega=0.85$, (e) $\omega=0.908$ and (f) $\omega=0.95$. The basins of attraction of the solutions depicted with black and blue dots are shown in orange and red colors, respectively. 
(a)
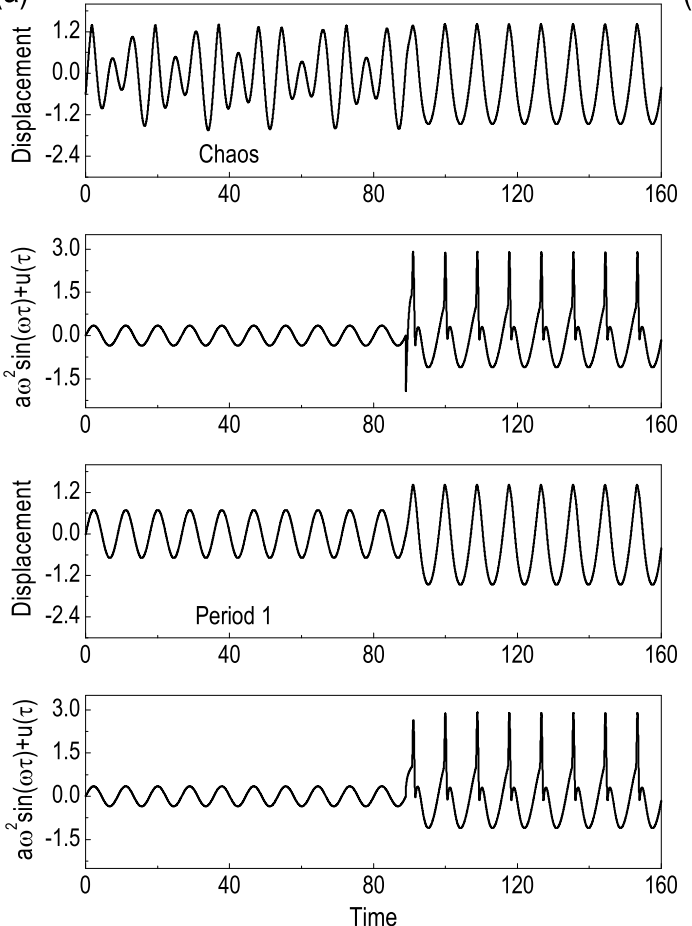

(c)

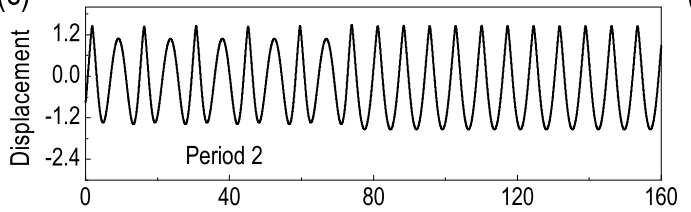

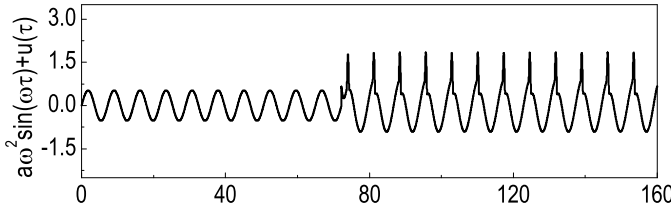
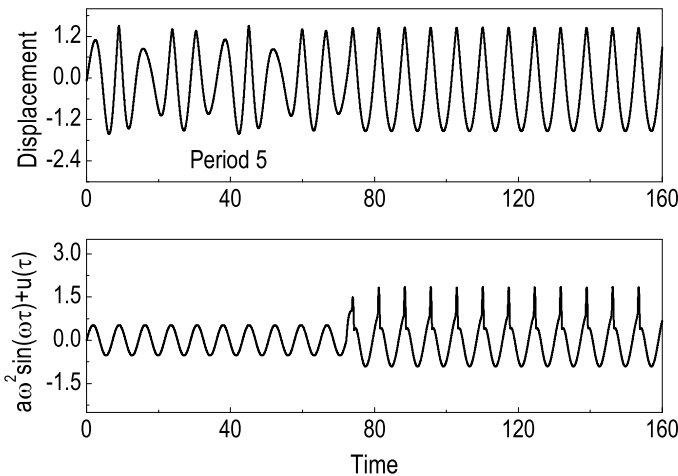

(b)
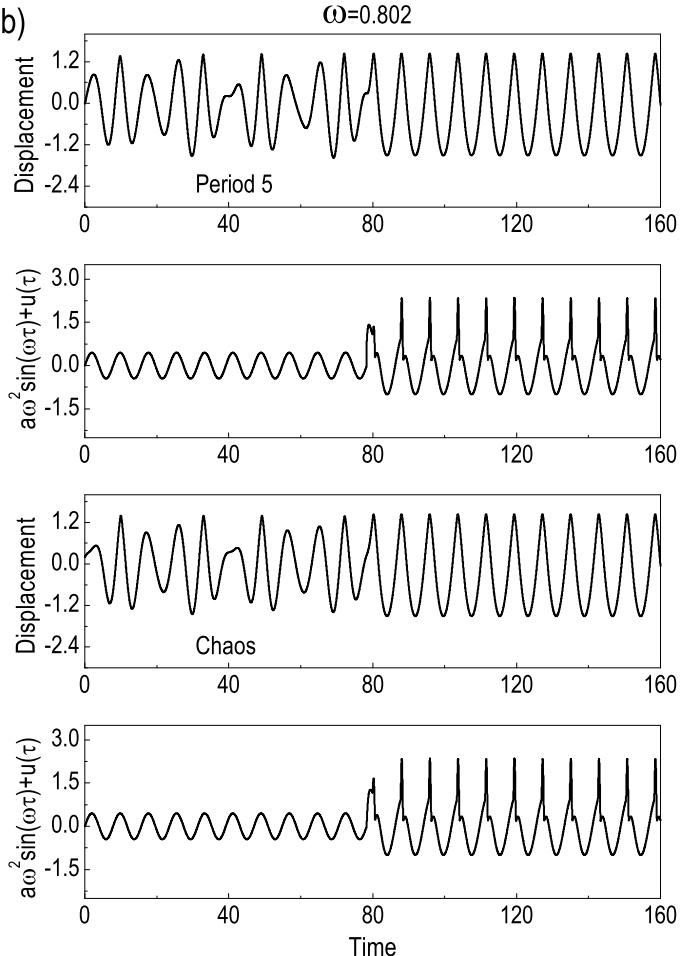

(d)
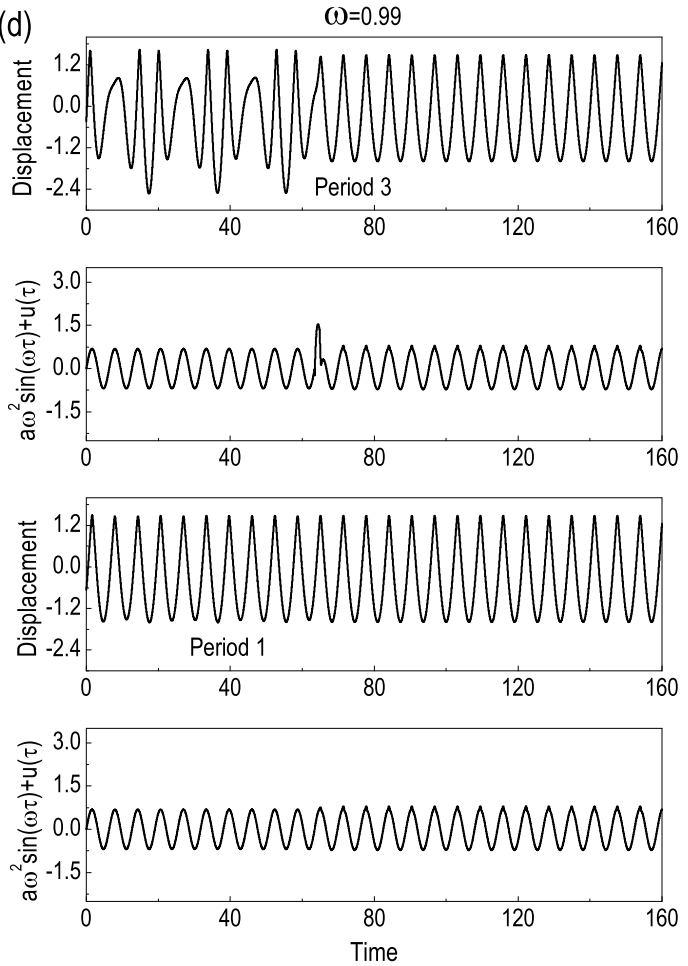

Figure 8: Trajectories and external excitations of the impact oscillator with the linear augmentation control inputs as a function of time, computed for $\zeta=0.01, e=1.26, a=0.7, \beta=28, \epsilon=2, \lambda=1$ and (a) $\omega=0.706$, (b) $\omega=0.802$, (c) $\omega=0.87$, (d) $\omega=0.99$. 
Previous studies have revealed that such peaks can be reduced by suitably choosing the time at which the controller is activated. This is, however, a matter that lies outside the scope of the present work and will be investigated in more detail in a future publication.

\subsection{Suppressing grazing-induced chaos}

In this section we will consider the scenario in which chaotic motion and periodic behavior coexist for the same set of parameter values. Such dynamical scenario is presented in Fig. 6, where a bifurcation diagram has been generated by following two coexisting solutions as the excitation frequency $\omega$ varies. Our numerical investigation reveals that chaotic attractors due to grazing events can be found in the parameter windows $\omega \in[0.704,0.707], \omega \in[0.801,0.805]$ and $\omega \in[0.893,0.914]$. The evolution of the basins of attraction of the impact oscillator is depicted in Fig. 7, for some selected values of $\omega$. In these pictures, it can be verified that the system indeed evolves from monostability to bistability as the excitation frequency is changed. Furthermore, it can be observed that for some frequency values chaotic and periodic behavior coexist, and the main purpose in this case will be to suppress such chaotic responses by driving the system to a suitably chosen periodic solution, for the parameter window $\omega \in[0.7,1.0]$.

In order to achieve the goal outlined in the previous paragraph, we will choose a reference solution $\left(x_{d}(\tau), v_{d}(\tau)\right), \tau \geq 0$, as a period-1 response of the impact oscillator with one impact period for $\omega=1$, corresponding to the right end of the black branch shown in Fig. 6. With this reference solution fixed, we will apply the linear augmented controller in order to drive the system to a period-1 response. The result can be seen in Fig. 8, which presents time histories of the mass position of the impact oscillator and the external excitation resulting from the sinusoidal forcing and the control signal $u$. Here, the controller is switched on after eleven periods of sinusoidal excitation, and in all cases the system response was successfully driven to the desired period-1 behavior with one impact per period, both for the cases where the system was operating under undesired periodic modes or chaotic motion. Consider for instance the case shown in panel (b) of Fig. 8, for $\omega=0.802$. The first two plots correspond to the situation for which the system is operating under a period-5 motion at the beginning, while the two lower figures resembles a similar situation where the initial state is in this case a chaotic response. After the controller is switched on, it can be observed that in both cases the system settles down to the same period-1 motion, once transients have decayed. This pattern can be observed in all scenarios displayed in Fig. 8, which confirms the effectiveness of the linear augmented controller for the considered cases. Fig. 9 presents the evolution of the desired period-1 solutions of the impact oscillator obtained by applying the linear augmented control law, as the frequency of excitation varies. In this picture we can verify that the target period-1 solution persists over the whole parameter window $\omega \in[0.7,1.0]$.

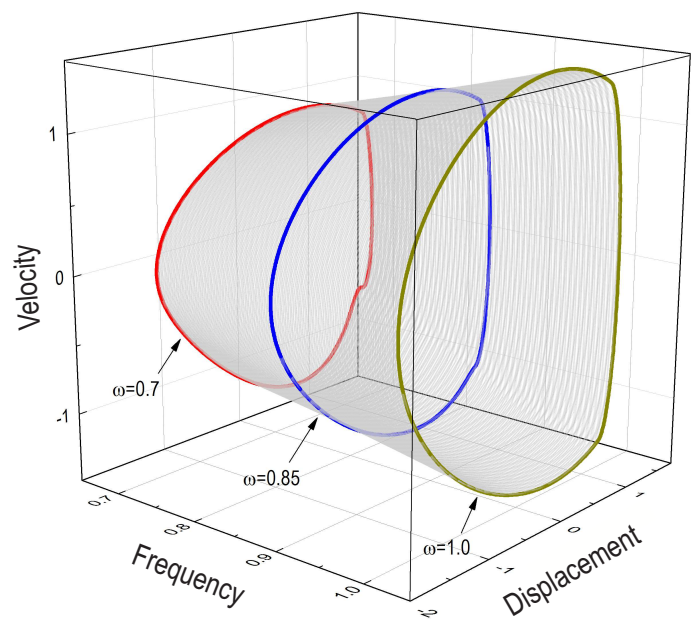

Figure 9: Periodic orbits of the impact oscillator with the linear augmentation control law under variation of excitation frequency $\omega \in[0.7,1.0]$, computed for $\zeta=0.01, e=1.26, a=0.7, \beta=28, \epsilon=2$ and $\lambda=1$. 


\section{Performance analysis of the linear augmentation control law}

One of the main purposes of a control method is to enable the user to regulate the transient and steady-state response of a system. However, these regulation capabilities are very often achieved at a certain cost, in terms of power consumption, computational effort, operational costs, etc. From a practical point of view, it is useful to define certain performance indices that allow the user to measure how well the control scheme carries out the tasks for which it was designed. Furthermore, the system specifications are usually given precisely in terms of two or more performance indices, which in many cases need to be adjusted so as to effect a compromise, since optimizing one performance index may lead to a detrimental value of another. Therefore, a control method is considered to be optimum when the control parameters are adjusted so that a performance index reaches an extremum (usually a minimum value), while other indices are kept within predefined suitable boundaries. In the present section we will show how the proposed linear augmentation control law can be studied via numerical continuation techniques in order to determine system parameters yielding an optimal control scheme.

(b)

(a)
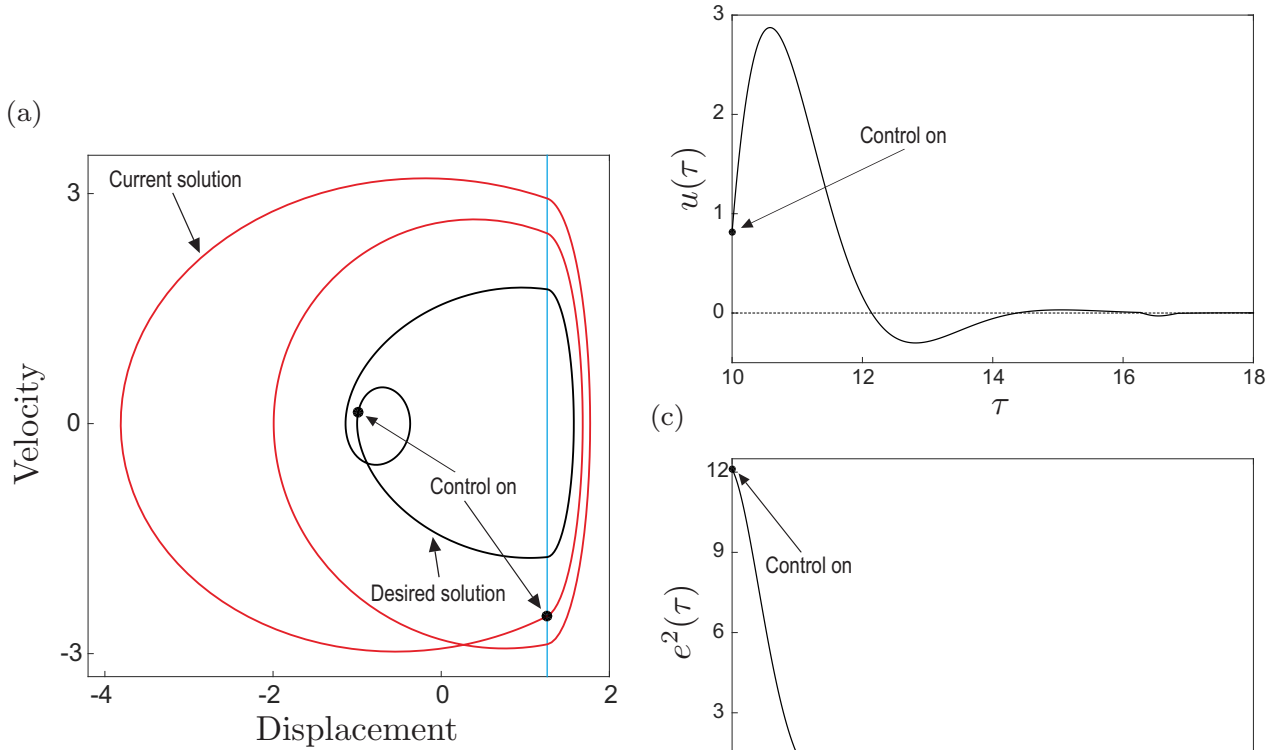

(c)

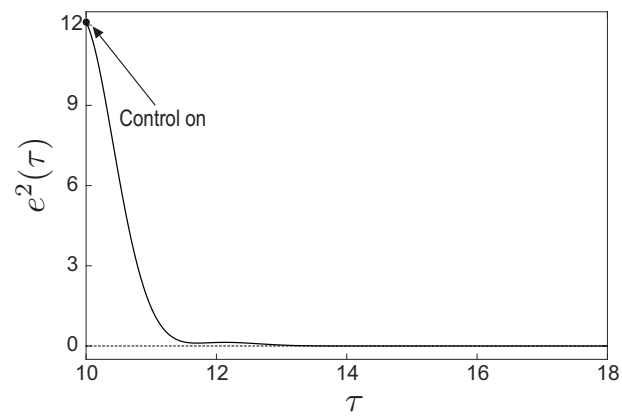

Figure 10: (a) Coexisting attractors of system (6) computed for the parameter values $\omega=0.69, a=2.21, \zeta=0.01, e=1.26$ and $\beta=28$ (without control $\epsilon=\lambda=0$ ). The dots indicate the instant at which the control is switched on $\left(\right.$ at $T_{0}=10$ ). Panels (b) and (c) show the behavior of the control signal $u(\tau)$ and the quadratic error $e^{2}(\tau)$, respectively, after the control is turned on, with $\epsilon=2$ and $\lambda=1$. In this implementation, the system response is switched from the current solution (red attractor) to the desired orbit (black attractor).

\subsection{Preliminary setup}

In the present section we will consider the case in which the linear augmentation control law is applied to induce a transition from the red (current) to the black (desired) periodic solution shown in Fig. 10(a), which coexist at the same parameter values. This scenario is motivated by the fact that for the desired attractor, the average power consumed per period by the impact oscillator is significantly less than for the red periodic solution, see Fig. 2(b). In order to investigate the performance of the control law for this case we will apply numerical continuation methods implemented by the continuation software COCO [34], an analysis and development platform for the numerical solution of continuation problems in MATLAB.

A remarkable characteristic of $\mathrm{COCO}$ is that it provides the user with a general-purpose computational platform based on the extended continuation paradigm [35]. The key idea of continuation is, in the 
simplest form, to approximate a one-dimensional branch of solutions of a (nonlinear) system $F(z)=0$ (referred to as a zero problem) of $n$ equations in $n+1$ unknowns, in the context of the Implicit Function Theorem [36]. In many practical problems, it may be useful to monitor the value of certain test functions along a particular solution branch during the continuation process. In our case, such functions will provide relevant information regarding the performance of the control method considered in the present work (see below). The main idea of the extended continuation paradigm is to embed the test functions and the system $F(z)=0$ in a zero problem of larger dimension. This approach offers great flexibility for detecting special points on a solution branch and then tracing a locus of the same by allowing an additional continuation parameter to vary, in the case when the system $F(z)=0$ has more than $n+1$ unknowns. By means of the COCO feature described above, we will carry out a detailed investigation of the performance of the control method studied in the present work. Specifically, we will make use of the COCO-toolbox 'hspo', which enables the numerical continuation and bifurcation detection of periodic orbits of non-smooth dynamical systems. One of the main features of this toolbox is that it implements a segment-specific discretization strategy in the framework of multisegment boundary-value problems [34].

The control scenario to be considered in this section involves the computation of two coexisting periodic solutions, the current and the desired ones, as shown in Fig. 10(a). Therefore, it will be convenient to consider the following augmented system for the numerical computations

$$
\left\{\begin{array}{l}
x^{\prime}(\tau)=v(\tau) \\
v^{\prime}(\tau)=a \omega^{2} \sin (\omega \tau)+u(\tau)-2 \zeta v(\tau)-x(\tau)-\beta(x(\tau)-e) H(x(\tau)-e), \\
x_{d}^{\prime}(\tau)=v_{d}(\tau) \\
v_{d}^{\prime}(\tau)=a \omega^{2} \sin (\omega \tau)-2 \zeta v_{d}(\tau)-x_{d}(\tau)-\beta\left(x_{d}(\tau)-e\right) H\left(x_{d}(\tau)-e\right),
\end{array}\right.
$$

with $u(\tau)=\epsilon\left[\left(x_{d}(\tau)-x(\tau)\right)+\lambda\left(v_{d}(\tau)-v(\tau)\right)\right]$, for $\tau \geq 0$. As before, $(x(\tau), v(\tau))$ and $\left(x_{d}(\tau), v_{d}(\tau)\right)$ stand for the current and desired trajectories of the system, respectively. The numerical approach will consist in performing the continuation of the current and desired solutions simultaneously, computed from the augmented system (6) for $\epsilon=\lambda=0$. During the continuation we will monitor the values of some predefined performance indices (see below), as outlined before, with $\epsilon \neq 0$ and $\lambda \neq 0$, which can be readily implemented in COCO due to the possibility of introducing user-defined monitor functions. In this way, the numerical scheme is divided into two separate tasks that are run simultaneously, that is, the continuation of the current and desired solutions (with $\epsilon=\lambda=0$ ) and the study of the transient behavior when the control law is switched on (with $\epsilon \neq 0$ and $\lambda \neq 0$ ), via the performance indices defined as monitor functions in COCO.

In order to investigate the performance of the control method, we will introduce the error function

$$
e^{2}(\tau)=\left(x_{d}(\tau)-x(\tau)\right)^{2}+\left(v_{d}(\tau)-v(\tau)\right)^{2}, \quad \tau \geq 0
$$

which will be used to measure the distance between the current and desired solutions during the transient phase, after the control has been activated. Thus, in our study the following performance indices will be considered

$$
E_{u}=\int_{T_{0}}^{T_{f}} u(\tau)^{2} d \tau, \quad S_{e^{2}}=\int_{T_{0}}^{T_{f}}\left(\tau-T_{0}\right) e^{2}(\tau) d \tau,
$$

where $T_{0}$ represents the time at which the control law is activated, while the upper limit $T_{f}>T_{0}$ is a given final time that allow the transients to decay. In our investigation, we will choose $T_{f}$ so that $T_{f}-T_{0}=10 \cdot \frac{2 \pi}{\omega}$, i.e., the measures in $(7)$ will be computed over the first 10 periods of external excitation after the control scheme has been switched on. The first index $E_{u}$ represents the expenditure of energy due to the control signal $u(\tau)$, for $\tau \in\left[T_{0}, T_{f}\right]$, and in many applications this is considered as a cost that should be kept as low as possible. The second index $S_{e^{2}}$ is referred in the literature as the integral of time multiplied by the squared error [37], which we will abbreviate as integral quadratic error. This index measures the system's performance in terms of the behavior of the error during the transient phase, after the control is activated. An important feature of this index is that it pays less attention to possible large 
initial errors, while it penalizes transients with long duration. In the next section we will investigate the behavior of the performance indices defined in (7) when the system parameters are varied. In particular, we will use numerical continuation techniques via the platform COCO to determine parameter values yielding an optimal control scheme.

(a)

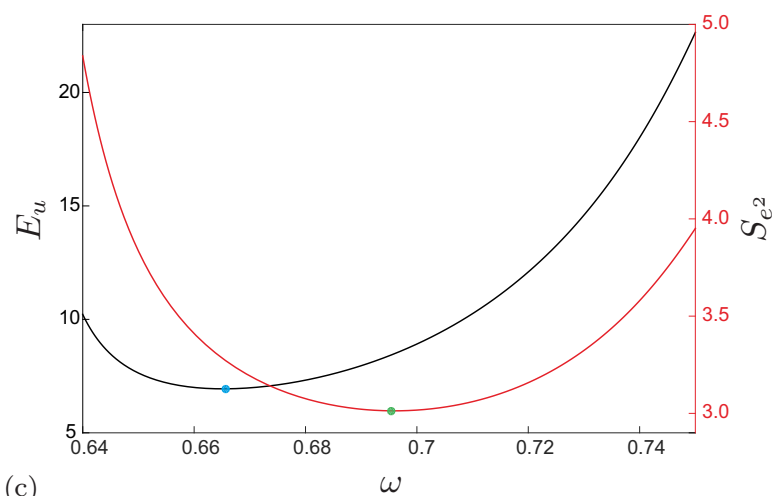

(c)



(e)

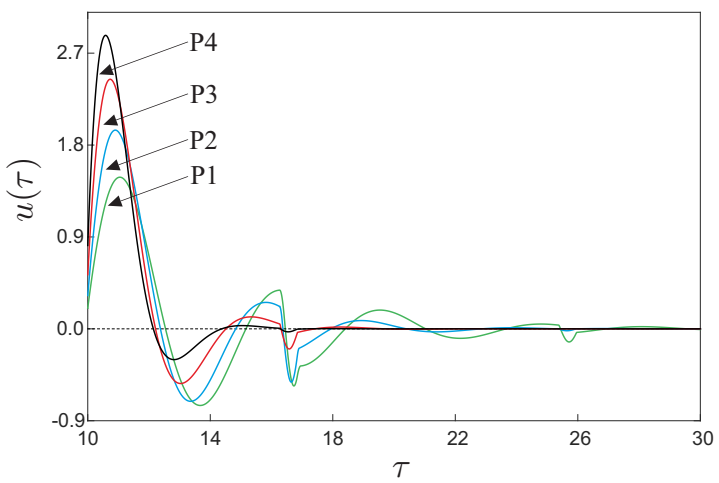

(b)
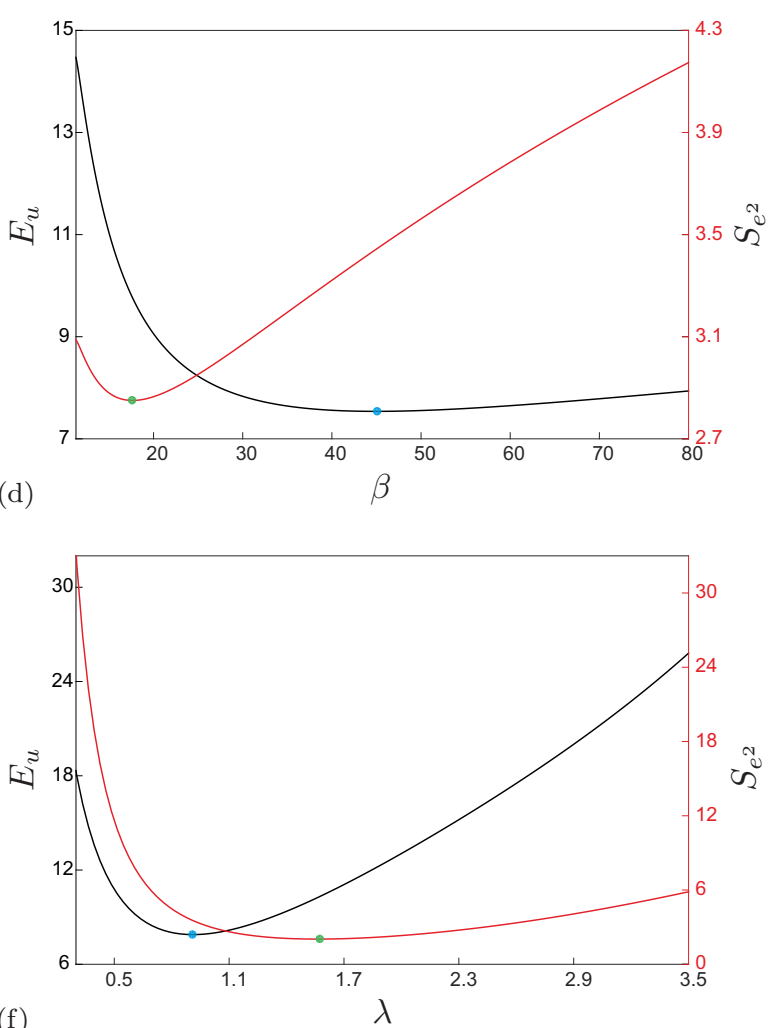

(f)

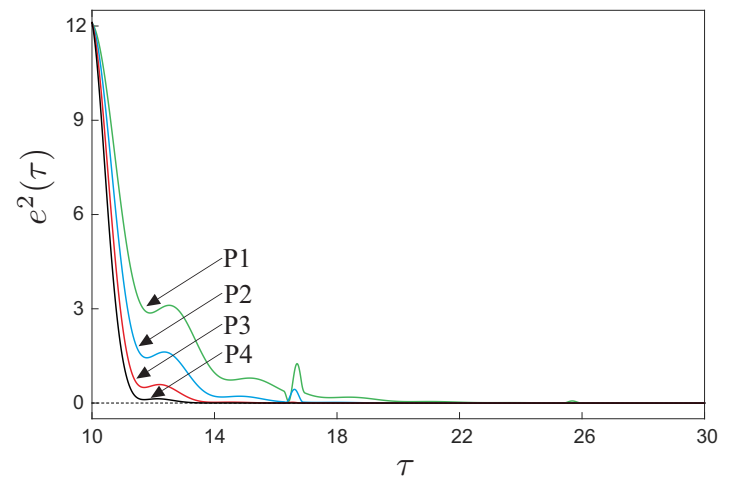

Figure 11: Numerical continuation of the dynamic response of system (6), computed for the parameter values indicated in Fig. 10. The panels show the behavior of the energy $E_{u}$ (black curve) and the integral quadratic error $S_{e^{2}}$ (red curve) with respect to (a) $\omega$, (b) $\beta$, (c) $\epsilon$ and (d) $\lambda$. Panels (e) and (f) show the time series of the control signal $u(\tau)$ and the quadratic error $e^{2}(\tau)$, respectively, for the points P1-P4 shown in panel (c). In panels (a), (b) and (d) the blue and green dots denote parameter values at which the energy $E_{u}$ and the integral quadratic error $S_{e^{2}}$ attain a local minimum, respectively.

\subsection{Optimization of the control scheme via continuation methods}

Our main concern in this section will be to study in detail the behavior of the performance indices introduced before with respect to parameter variations. In Fig. 11 we show the numerical continuation of the dynamic response of system (6) with respect to the parameters, $\omega, \beta, \epsilon$, and $\lambda$. During each 
continuation step, the performance indices defined in (7) are calculated and their behavior can be seen in panels (a) to (d). Here, points of optimal operation are detected, which are plotted in blue, when the energy $E_{u}$ is minimized, and in green, when the integral quadratic error $S_{e^{2}}$ is minimized. For the cases where $\omega, \beta$ and $\lambda$ are adjusted, it can be observed that, when one performance index is minimized, the other one achieves values above its optimum, which reveals that there is a compromise between the measures $E_{u}$ and $S_{e^{2}}$. This compromise is even more noticeable when the coefficient $\epsilon$ is varied. As can be seen in Fig. 11(c), the energy of the control signal and the integral quadratic error vary monotonically when the parameter increases. Specifically, when $\epsilon$ increases, the expenditure of energy for control grows, while the integral quadratic error reduces. This can be verified in Figs. 11(e) and (f), where the time series of the control signal $u(\tau)$ and the quadratic error $e^{2}(\tau), \tau \geq 0$, are presented. Here, it can be seen that the smaller $\epsilon$, the longer the transient phase takes before the error decays, and the smaller the energy spent for control. However, this apparent correlation between energy expenditure for control and duration of the transients need not be true when other parameters are adjusted. For instance, in Fig. 11(a) we can see that after the integral quadratic error has reached a minimum (at $\omega \approx 0.6954$ ), an increment in the energy $E_{u}$ does not lead to a reduction in the error, meaning that there may be an inefficient use of energy in the system. This motivates a further numerical study of the behavior of the performance indices $E_{u}$ and $S_{e^{2}}$, using path-following techniques, as detailed below.

Fig. 12 shows the result of the two-parameter continuation of the dynamic response of system (6) with respect to the control parameters $\epsilon$ and $\lambda$. Specifically, using the continuation platform COCO, we computed a number of curves in the $\epsilon-\lambda$ plane for which the condition $E_{u}=K$ is imposed, for various fixed values of $K$. During the continuation process, we monitored the behavior of the integral quadratic error $S_{e^{2}}$, which is shown in Fig. 12(a). In this way, we could determine a point in the $\epsilon-\lambda$ plane at which $S_{e^{2}}$ attains the minimum value under the restriction $E_{u}=K$. Therefore, depending on the system specifications regarding energy consumption, we can determine the control parameters yielding an optimal control scheme. Fig. 12(b) shows how the optimal control point varies when the fixed condition $E_{u}=K$ is changed.

(a)

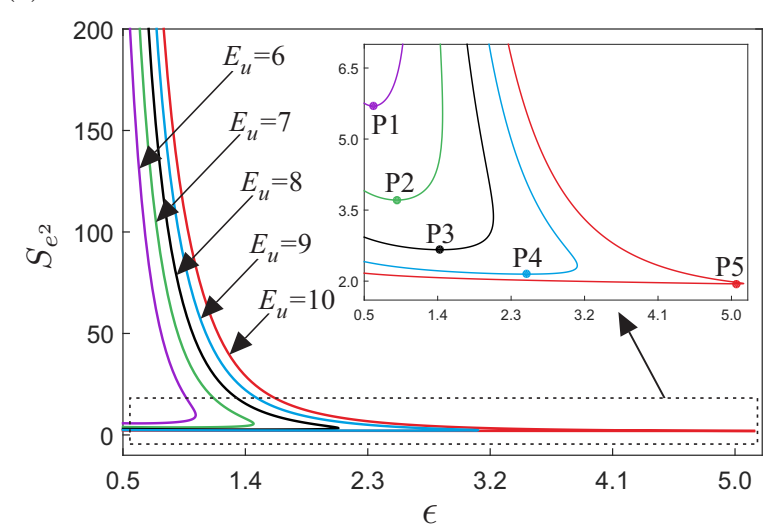

(b)

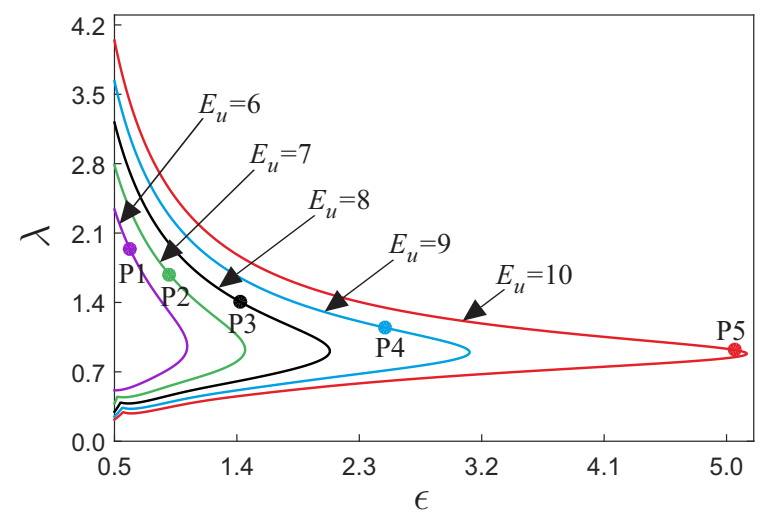

Figure 12: Two-parameter continuation of the dynamic response of system (6) with respect to $\epsilon$ and $\lambda$, keeping the energy $E_{u}$ constant. Panel (a) shows the behavior of the integral quadratic error $S_{e^{2}}$ for fixed $E_{u}=6,7,8,9$ and 10 . The inner set depicts an enlargement of the boxed region. In this diagram, points for which the integral quadratic error attains a minimum value are labeled $\mathrm{P} 1(\epsilon \approx 0.6148, \lambda \approx 1.9357), \mathrm{P} 2(\epsilon \approx 0.9029, \lambda \approx 1.6839), \mathrm{P} 3(\epsilon \approx 1.4276, \lambda \approx 1.4035), \mathrm{P} 4$ $(\epsilon \approx 2.4871, \lambda \approx 1.1465)$ and $\mathrm{P} 5(\epsilon \approx 5.0599, \lambda \approx 0.9209)$. Panel (b) shows a family of curves on the $\epsilon-\lambda$ plane for which the energy $E_{u}$ remains constant. The points P1-P5 correspond to the optimal operation points depicted in panel (a).

\section{Conclusions}

This paper presents a numerical study of a recently developed control method based on linear augmentation. Our study considers a particular case where the control scheme is applied to an impacting system modeled by a non-smooth dynamical system exhibiting multistability. The main objective in 
this application is to drive the impacting system to a desired periodic solution via the linear augmented controller. This approach allows us to address two control issues that are common to a wide range of engineering applications, namely, switching between coexisting attractors without altering the system's parameters and suppressing undesired chaotic responses. The first control issue is motivated by a dynamical scenario where the impacting system is bistable, with two coexisting periodic solutions for which there is a significant difference in the power required to operate the system. Specifically, the linear augmentation control law is used to drive the system from the periodic regime with high energy consumption to a periodic response with a much lower power dissipation. The second control issue is investigated for a parameter range in which chaotic and periodic behavior coexist in the impacting system. In this case, the controller is applied in such a way that the system can switch from a grazing-induced chaotic attractor to a suitably chosen periodic solution, which is a common problem in many engineering applications.

The effectiveness of the proposed control scheme is studied by a number of numerical techniques. For this purpose, bifurcation diagrams and basins of attraction are computed for various dynamical scenarios with a practical relevance. Furthermore, the performance of the linear augmentation control law is analyzed in terms of integral indices related to the expenditure of energy due to the control signals and the behavior of the error during the transient phase after the controller is activated. To study in detail the effect of the system and control parameters on these performance indices, numerical continuation methods for non-smooth dynamical systems were applied via the continuation platform COCO [34]. Our numerical study revealed a compromise between the performance indices when the parameters are adjusted. Specifically, optimizing one index may lead to unsuitable values of the other, as is common in many practical cases. To investigate this issue further, we used COCO's capabilities to introduce parameter constraints during continuation in order to generate a family of curves in a two-dimensional space defined by the main control parameters. In this way, we were able to systematically determine parameter values yielding optimal control for given energy constraints, and one path of future research may be the implementation of the proposed optimization scheme to more involved mechanical systems and the experimental verification of the numerical predictions.

\section{Acknowledgements}

The second author has been supported by the 'DRESDEN Fellowship Programm' of the TU Dresden.

\section{References}

[1] J.-H. Ho, V.-D. Nguyen, and K.-C. Woo, "Nonlinear dynamics of a new electro-vibro-impact system," Nonlinear Dynamics, vol. 63, pp. 35-49, 2011.

[2] E. E. Pavlovskaia, D. C. Hendry, and M. Wiercigroch, "Modelling of high frequency vibro-impact drilling," Internat. J. of Mech. Sci., vol. 91, pp. 110-119, 2015.

[3] Y. Liu, E. E. Pavlovskaia, and M. Wiercigroch, "Experimental verification of the vibro-impact capsule model," Nonlinear Dynamics, vol. 83, no. 1, pp. 1029-1041, 2016.

[4] J. Páez Chávez, Y. Liu, E. E. Pavlovskaia, and M. Wiercigroch, "Path-Following Analysis of the Dynamical Response of a Piecewise-Linear Capsule System," Commun. Nonlinear Sci. Numer. Simul., vol. 37, pp. 102-114, 2016.

[5] S. L. T. de Souza, I. L. Caldas, R. L. Viana, and J. M. Balthazar, "Control and chaos for vibro-impact and non-ideal oscillators," J. Theor. Appl. Mech., vol. 46, no. 3, pp. 641-664, 2008.

[6] S. Lahriri, I. F. Santos, H. I. Weber, and H. J. Hartmann, "On the nonlinear dynamics of two types of backup bearings - Theoretical and experimental aspects," J. Eng. Gas Turb. Power, vol. 134, no. $11,2012$.

[7] J. Páez Chávez, V. Vaziri Hamaneh, and M. Wiercigroch, "Modelling and Experimental Verification of an Asymmetric Jeffcott Rotor with Radial Clearance," J. Sound Vibration, vol. 334, pp. 86-97, 2015 . 
[8] Y. Liu, M. Wiercigroch, J. Ing, and E. E. Pavlovskaia, "Intermittent control of coexisting attractors," Philos. Trans. R. Soc. Lond., Ser. A, Math. Phys. Eng. Sci., vol. 371, no. 1993, p. 20120428, 2013. (15 pages).

[9] Y. Liu, E. E. Pavlovskaia, M. Wiercigroch, and Z. K. Peng, "Forward and backward motion control of a vibro-impact capsule system," Int. J. Non-Linear Mech., vol. 70, pp. 30-46, 2015.

[10] Y. Liu and J. Páez Chávez, "Controlling multistability in a vibro-impact capsule system," Nonlinear Dynamics, 2017. Article in press.

[11] O. K. Ajibose, M. Wiercigroch, E. E. Pavlovskaia, and A. R. Akisanya, "Global and local dynamics of drifting oscillator for different contact force models," Int. J. Non-Linear Mech., vol. 45, no. 9, pp. $850-858,2010$.

[12] E. M. Navarro-López, "An alternative characterization of bit-sticking phenomena in a multi-degreeof-freedom controlled drillstring," Nonlinear Anal. Real World Appl., vol. 10, no. 5, pp. 3162-3174, 2009.

[13] G. A. Leonov, N. V. Kuznetsov, M. A. Kiseleva, E. P. Solovyeva, and A. M. Zaretskiy, "Hidden oscillations in mathematical model of drilling system actuated by induction motor with a wound rotor," Nonlinear Dynamics, vol. 77, no. 1, pp. 277-288, 2014.

[14] M. Kapitaniak, V. Vaziri Hamaneh, J. Páez Chávez, K. Nandakumar, and M. Wiercigroch, "Unveiling complexity of drill-string vibrations: Experiments and modelling," Internat. J. of Mech. Sci., vol. 101-102, pp. 324-337, 2015.

[15] J. Páez Chávez and M. Wiercigroch, "Bifurcation Analysis of Periodic Orbits of a Non-Smooth Jeffcott Rotor Model," Commun. Nonlinear Sci. Numer. Simul., vol. 18, no. 9, pp. 2571-2580, 2013.

[16] B. Blażejczyk-Okolewska and T. Kapitaniak, "Co-existing attractors of impact oscillator," Chaos, Solitons and Fractals, vol. 9, no. 8, pp. 1439-1443, 1998.

[17] O. Makarenkov and J. S. W. Lamb, "Dynamics and bifurcations of nonsmooth systems: A survey," Physica D, vol. 241, no. 22, pp. 1826-1844, 2012.

[18] S. Kryzhevich and M. Wiercigroch, "Topology of vibro-impact systems in the neighborhood of grazing," Physica D, vol. 241, no. 22, pp. 1919-1931, 2012.

[19] H. Jiang and M. Wiercigroch, "Geometrical insight into non-smooth bifurcations of a soft impact oscillator," IMA J. Appl. Math., 2016. Article in press.

[20] J. Páez Chávez, E. E. Pavlovskaia, and M. Wiercigroch, "Bifurcation analysis of a piecewise-linear impact oscillator with drift," Nonlinear Dynamics, vol. 77, no. 1-2, pp. 213-227, 2014.

[21] J. Ing, E. E. Pavlovskaia, M. Wiercigroch, and S. Banerjee, "Experimental study of impact oscillator with one-sided elastic constraint," Philos. Trans. R. Soc. Lond., Ser. A, Math. Phys. Eng. Sci., vol. 366, no. 1866, pp. 679-704, 2008.

[22] J. Ing, E. E. Pavlovskaia, M. Wiercigroch, and S. Banerjee, "Bifurcation analysis of an impact oscillator with a one-sided elastic constraint near grazing," Physica D, vol. 239, no. 6, pp. 312-321, 2010.

[23] S. Kundu, S. Banerjee, J. Ing, E. E. Pavlovskaia, and M. Wiercigroch, "Singularities in soft-impacting systems," Physica D, vol. 241, no. 5, pp. 553-565, 2012.

[24] M. Liao, J. Ing, J. Páez Chávez, and M. Wiercigroch, "Bifurcation Techniques for Stiffness Identification of an Impact Oscillator," Commun. Nonlinear Sci. Numer. Simul., vol. 41, pp. 19-31, 2016. 
[25] E. E. Pavlovskaia, J. Ing, M. Wiercigroch, and S. Banerjee, "Complex dynamics of bilinear oscillator close to grazing," Internat. J. of Bif. and Chaos, vol. 20, no. 11, pp. 3801-3817, 2010.

[26] S. L. T. de Souza and I. L. Caldas, "Controlling chaotic orbits in mechanical systems with impacts," Chaos, Solitons and Fractals, vol. 19, no. 1, pp. 171-178, 2004.

[27] S. L. T. de Souza, I. L. Caldas, and R. L. Viana, "Damping control law for a chaotic impact oscillator," Chaos, Solitons and Fractals, vol. 32, no. 2, pp. 745-750, 2007.

[28] H. Dankowicz and J. Jerrelind, "Control of near-grazing dynamics in impact oscillators," Proc. R. Soc. A, vol. 461, no. 2063, pp. 3365-3380, 2005.

[29] R. Karnatak, "Linear augmentation for stabilizing stationary solutions: Potential pitfalls and their application," PLoS ONE, vol. 10, no. 11, p. e0142238, 2015. (22 pages).

[30] P. R. Sharma, A. Sharma, M. D. Shrimali, and P. Prasad, "Targeting fixed-point solutions in nonlinear oscillators through linear augmentation," Physical Review E, vol. 83, p. 067201, 2011. (4 pages).

[31] P. R. Sharma, M. D. Shrimali, A. Prasad, and U. Feudel, "Controlling bistability by linear augmentation," Phys. Lett. A, vol. 377, no. 37, pp. 2329-2332, 2013.

[32] P. R. Sharma, A. Singh, P. Prasad, and M. D. Shrimali, "Controlling dynamical behavior of driveresponse system through linear augmentation," Eur. Phys. J. Spec. Top., vol. 223, no. 8, pp. 15311539, 2014.

[33] P. R. Sharma, M. D. Shrimali, A. Prasad, N. V. Kuznetsov, and G. A. Leonov, "Controlling dynamics of hidden attractors," Internat. J. of Bif. and Chaos, vol. 25, no. 4, p. 1550061, 2015. (7 pages).

[34] H. Dankowicz and F. Schilder, Recipes for continuation. Computational Science and Engineering, Philadelphia: SIAM, 2013.

[35] H. Dankowicz and F. Schilder, "An extended continuation problem for bifurcation analysis in the presence of constraints," J. Comput. Nonlin. Dyn., vol. 6, no. 3, p. 031003, 2011. (8 pages).

[36] B. Krauskopf, H. Osinga, and J. Galán-Vioque, eds., Numerical Continuation Methods for Dynamical Systems. Understanding Complex Systems, Netherlands: Springer-Verlag, 2007.

[37] R. C. Dorf and R. H. Bishop, Modern control systems. New Jersey: Prentice Hall, twelfth ed., 2011. 